\title{
Urinary Tract Infection among Pregnant Women at Pumwani Maternity Hospital, Nairobi, Kenya: Bacterial Etiologic Agents, Antimicrobial Susceptibility Profiles and Associated Risk Factors
}

\author{
Hellen A. Onyango ${ }^{1}$, Carolyne Ngugi ${ }^{2}$, John Maina ${ }^{1}$, John Kiiru ${ }^{3}$ \\ ${ }^{1}$ Department of Medical Microbiology, Jomo Kenyatta University of Agriculture and Technology, Nairobi, Kenya \\ ${ }^{2}$ Department of Medical Microbiology, College of Health Sciences, Jomo Kenyatta University of Agriculture and Technology, \\ Nairobi, Kenya \\ ${ }^{3}$ Centre of Microbiology Research, Kenya Medical Research Institute, Nairobi, Kenya \\ Email: ^honyango79@gmail.com
}

How to cite this paper: Onyango, H.A., Ngugi, C., Maina, J. and Kiiru, J. (2018) Urinary Tract Infection among Pregnant Women at Pumwani Maternity Hospital, Nairobi, Kenya: Bacterial Etiologic Agents, Antimicrobial Susceptibility Profiles and Associated Risk Factors. Advances in Microbiology, 8, 175-187.

https://doi.org/10.4236/aim.2018.83012

Received: January 10, 2018

Accepted: March 18, 2018

Published: March 21, 2018

Copyright $(9) 2018$ by authors and Scientific Research Publishing Inc. This work is licensed under the Creative Commons Attribution International License (CC BY 4.0).

http://creativecommons.org/licenses/by/4.0/

\begin{abstract}
Urinary Tract Infections (UTIs) during pregnancy are among the most common infections worldwide and can lead to poor perinatal and maternal outcomes. This study determined the prevalence of UTIs during pregnancy, associated risk factors and antimicrobial susceptibility profiles of associated bacterial pathogens. A cross-sectional study was conducted among 210 pregnant women attending antenatal clinic at Pumwani Maternity hospital. Their social-demographic profiles were obtained using a structured questionnaire. Cultures were done from midstream urine and antimicrobial susceptibility testing determined using the disc diffusion test. The overall prevalence of UTI was $15.7 \%$ regardless of the women's age, parity and stage of gestation. Prevalence of asymptomatic and symptomatic bacteriuria was $4.3 \%$ and $11.4 \%$ respectively. Material of undergarment and frequency of changing the undergarments were found to contribute significantly to the acquisition of UTI $(\mathrm{P}<$ 0.05). E. coli was the most predominant UTI organism at (44.5\%) followed by K. pneumoniae (21.2\%) and S. aureus (15.1\%). Almost a half (over $49 \%$ ) of all Gram-negative organisms showed resistance prevalence against third generation cephalosporins, fluoroquinolones, Sulfamethoxazole-Trimethoprim, Cefoxitin, Nitrofurantoin and Amoxicillin-clavulanic acid. Gram-positive strains were susceptible to Amoxicillin-clavulanic acid, Nitrofurantoin, Linezolid and Ofloxacin. The prevalence of multi-drug resistance (MDR) in all study isolates was $96 \%$. Our data suggest a serious resistance trend among UTI strains and
\end{abstract}


more should be done to slow down this trend. Pregnant women should be screened by urine culture and treatment be guided by the antimicrobial susceptibility data.

\section{Keywords}

Urinary Tract Infection, Pregnancy, Risk Factors, Antimicrobial Resistance, ESBL, MRSA, MDR

\section{Introduction}

In developing countries, UTIs are among the most common health problems affecting women in their reproductive ages. Pregnant women are more susceptible to UTIs due to a combination of hormonal and physiologic changes that predispose them to bacteriuria [1]. The incidence of acute pyelonephritis in pregnant women is also significantly increased [2]. Factors such as history of recurrent urinary tract infection, diabetes, low social economic status, increasing maternal age, multiparity, and anatomical abnormalities of the urinary tract have also been associated with a two fold increase in bacteriuria during pregnancy [2], but the risk factors associated with UTIs in Africa remains poorly investigated.

Globally, the prevalence of UTI in pregnancy ranges between $13 \%-33 \%$ with symptomatic bacteriuria occurring in $1 \%-18 \%$ while asymptomatic cases are noted in $2 \%-10 \%$ of women [3]. The prevalence has remained constant and most of the recent observational studies, including those from developing countries, report almost similar rates [2]. The most common agent implicated in symptomatic and asymptomatic bacteriuria is Escherichia coli that is responsible for 70\% - 80\% of the infections [4]. Other microorganisms include Staphylococcus spp., Klebsiella pneumoniae, Proteus spp., Pseudomonas aeruginosa, Enterococcus spp. and Acinetobacter [5]. Past studies indicate that UTI among pregnant mothers in Kenya range from 10\% - 19\% [6]. However, most of these studies concentrate on selected bacterial pathogens and the larger spectrum of bacterial etiologic agents remains unknown. Furthermore, the socio-demographic and lifestyle factors associated with UTIs among pregnant women in Kenya remain uninvestigated.

In many hospitals in developing countries such as Kenya, routine urine culture is not carried out even for antenatal mothers. Currently, most patients are treated empirically without culture and antimicrobial susceptibility testing (AST) and treatment is therefore based on empiric guidelines that are rarely updated [7]. Even where UTI tests are done, only dipstick analysis and direct wet microscopy of urine are used, but these tests have poor positive and negative predictive values to detect bacteriuria particularly in asymptomatic persons [8]. We hypothesize that the overreliance on these methods and absence of culture and susceptibility testing have partially led to under-diagnosis of UTIs and this may be fueling the rising cases of treatment failure. In standard urine culture, a 
colony count of $10^{5} \mathrm{CFU} / \mathrm{ml}$ is usually considered significant for infection [8], but there's missing data regarding the phenotypic characteristics of isolates whose counts fall below this threshold. Currently, such counts are regarded as contaminants. It is therefore not known if such counts represent a receding infection or an infection that is establishing. In this study, we compared the results of antimicrobial resistance phenotypes from UTI cases and those normally regarded as contaminants.

Results of this study provide critical data to care givers and health planners regarding diagnosis, common etiological agents and probable treatment options with regard to antimicrobial resistance. This study also identified associated risk factors for UTI amongst pregnant women attending antenatal clinic at Pumwani Maternity Hospital (The largest antenatal clinic in Kenya).

\section{Materials and Methods}

\subsection{Study Site}

This was a cross sectional study carried out at Pumwani Maternity Hospital, an obstetric and referral hospital for expectant mothers in Nairobi, the Kenyan capital. The study targeted pregnant women seeking antenatal care services at the Hospital.

\subsection{Sampling Method}

Systematic random sampling technique was employed in recruiting participants until the expected study sample was attained. A structured questionnaire was used for gathering socio-demographic and lifestyle data from the participants. Participants were instructed on how to collect clean-catch midstream urine after signing the consent form. Each sample bottle had a date of collection and bar-code linked to the participant's questionnaire which was in turn linked to confidential patient information file.

\subsection{Urine Culture}

Urine aliquots of $10 \mu \mathrm{l}$ were plated directly on CLED, MacConkey and blood agar plates and incubated aerobically at $35^{\circ} \mathrm{C}-37^{\circ} \mathrm{C}$ for 24 hours. Colony counts yielding bacterial growth of $\geq 100,000\left(10^{5}\right) \mathrm{CFU} / \mathrm{ml}$ or more of pure isolates were deemed significant UTI cases whereas those yielding lower values were deemed as contaminants. At least 3 colonies of similar morphology per patient were randomly selected from UTI plates and purified before biochemical identification. Gram stain was done for all colonies analyzed and biochemical tests carried out to identify the isolates to the species level [9]. In order to investigate the profiles of the presumed contaminants colonies from at least $15 \%$ plates yielding CFUs below the $10^{5} \mathrm{CFU} / \mathrm{ml}$ thresholds were randomly selected and analyzed alongside those from UTI cases.

\subsection{Antimicrobial Susceptibility Testing}

Antimicrobial susceptibility testing of pure isolates was performed according to 
the Kirby-Bauer disc diffusion method [10] on Mueller Hinton agar (Oxoid, Ltd, England). The standard reference strains, S. aureus (ATCC-25923) and E. coli (ATCC-25922) were used as quality control organisms to ascertain antibiotic discs potency and quality of the test media. Results were interpreted based on CLSI 2016 guidelines [11]. The double disc synergy test was used to test for extended spectrum $\beta$-lactamase (ESBL) production following the CLSI (2015) guidelines. Isolates showing synergy zones between amoxicillin/clavulanic and one or more third generation cephalosporins were identified as ESBL-producers [12] Susceptibility to Cefoxitin was used for the detection of Methicillin Resistant Staphylococcus aureus-MRSA [13].

\subsection{Data Analysis}

Data analysis was done using using the SAS software, version 9.3 (SAS Institute). Chi-square test was applied for P-value derivation for socio-demographic and risk factors associated with UTIs. Binary logistic regression analysis was carried out to generate the adjusted odds ratio with $95 \%$ confidence interval, an alpha of less than $0.05(\mathrm{P}<0.05)$ was considered statistically significant.

\section{Results}

\subsection{Recruitment and Prevalence of UTI}

A total of 210 pregnant women were recruited in the study. The overall prevalence of UTI was $15.7 \%$. Prevalence of asymptomatic and symptomatic bacteriuria was $4.3 \%$ and $11.4 \%$ respectively.

\subsection{Social-Demographic Characteristics}

Pregnant mothers in the age group of 21 - 30 years had the highest prevalence of UTI at $75.8 \%$ followed by those aged $31-40$ years at $12.1 \%$, below 20 years of age at $9.1 \%$ while those above the age of 40 years had the least prevalence of $3 \%$. UTI prevalence was also high among mothers in the second trimester (60.6\%) compared to third trimester (24.2\%) and first trimester (15.2\%). High prevalence of bacteriuria was observed among multiparous mothers (72.7\%) as compared to nulliparous (27.3\%). Despite these variations, there was no significant association between UTI and maternal age, parity, occupation, gestation, marital status or level of education $\mathrm{P}>0.05$, Table 1 .

\subsection{Lifestyle Factors}

Among the lifestyle factors surveyed, mothers with multiple partners were two times more likely to develop a UTI (OR 2.45; P > 0.05) compared to those with a single partner Table 2 . Those who used non-cotton undergarments were three times more likely to develop a UTI compared to those who used cotton undergarments $(P=0.001)$. This study also found that the frequency of changing undergarment was significantly associated with UTI, $(\mathrm{P}<0.05)$. Those who changed once were 2 times likely to develop UTI compared to those who changed twice. 
When respondents were asked to state what causes UTI, a larger proportion (37.6\%) had no idea while others indicated that women got UTI from sexual intercourse (17.6\%), poor urogenital hygiene (9\%), contaminated toilets $(25.7 \%)$ and damp panties (10\%).

\subsection{Diversity of Bacterial Isolates from Urine}

From the 99 UTI isolates identified, 78 (78.8\%) were gram negative while 21 (21.2\%) were gram positive bacteria. E. coli was the most predominant UTI isolate at $44.5 \%$ followed by $K$. pneumoniae (21.2\%) and $S$. aureus (15.1\%). Other organisms included $P$. aeroginosa, Acinetobacter spp. and Enterococcus spp. each at $6.1 \%$. Analysis of $15 \%$ of the urine samples that would have been classified as

Table 1. Selected socio-demographic characteristics.

\begin{tabular}{|c|c|c|c|c|c|c|c|c|}
\hline \multirow{2}{*}{ Variables } & \multirow{2}{*}{ Response } & \multicolumn{2}{|c|}{ With UTI } & \multicolumn{2}{|c|}{ Without UTI } & \multirow{2}{*}{ OR } & \multirow{2}{*}{$95 \% \mathrm{CI}$} & \multirow{2}{*}{ P-value } \\
\hline & & $\mathrm{N}$ & $\%$ & $\mathbf{N}$ & $\%$ & & & \\
\hline Overall & & 33 & 15.7 & 177 & 84.3 & & & \\
\hline \multirow{2}{*}{ Blood Pressure } & Normal & 28 & 84.8 & 163 & 92.1 & 0.481 & $0.161-1.441$ & 0.183 \\
\hline & Abnormal & 5 & 15.2 & 14 & 7.9 & 2.079 & $0.694-6.227$ & 0.190 \\
\hline \multirow{4}{*}{ Maternal Age } & $\leq 20$ years & 3 & 9.1 & 18 & 10.2 & 1.132 & $0.314-4.084$ & 0.850 \\
\hline & 21 - 30 years & 25 & 75.8 & 134 & 75.7 & 0.997 & $0.419-2.373$ & 0.995 \\
\hline & 31 - 40 years & 4 & 12.1 & 22 & 12.4 & 1.029 & $0.330-3.207$ & 0.961 \\
\hline & 41 - 50 years & 1 & 3 & 3 & 1.7 & 0.552 & $0.056-5.472$ & 0.606 \\
\hline \multirow{2}{*}{ Parity } & Nulliparous & 9 & 27.3 & 64 & 36.2 & 1.510 & $0.662-3.447$ & 0.325 \\
\hline & Multiparous & 24 & 72.7 & 113 & 63.8 & 0.662 & $0.290-1.511$ & 0.317 \\
\hline \multirow{4}{*}{ Marital Status } & Single & 4 & 12.1 & 14 & 7.9 & 0.623 & $0.191-2.025$ & 0.428 \\
\hline & Married & 28 & 84.8 & 160 & 90.4 & 1.681 & $0.574-4.924$ & 0.339 \\
\hline & Divorced & 1 & 3 & 2 & 1.1 & 1.011 & $0.996-1.027$ & 0.540 \\
\hline & Widowed & 0 & 0 & 1 & 0.6 & 0.182 & $0.011-2.982$ & 0.181 \\
\hline \multirow{3}{*}{ Gestation } & $1^{\text {st }}$ Trimester & 5 & 15.2 & 43 & 24.3 & 1.797 & $0.653-4.942$ & 0.251 \\
\hline & $2^{\text {nd }}$ Trimester & 20 & 60.6 & 84 & 47.5 & 0.587 & $0.275-1.253$ & 0.165 \\
\hline & $3^{\text {rd }}$ Trimester & 8 & 24.2 & 50 & 28.2 & 1.230 & $0.520-2.910$ & 0.637 \\
\hline \multirow{2}{*}{ Occupation } & Employed & 15 & 45.5 & 107 & 60.5 & 1.834 & $0.868-3.877$ & 0.109 \\
\hline & Unemployed & 18 & 54.5 & 70 & 39.5 & 0.545 & $0.258-1.152$ & 0.111 \\
\hline \multirow{3}{*}{ Level of Education } & Primary & 10 & 30.3 & 55 & 31.1 & 1.037 & $0.462-2.326$ & 0.930 \\
\hline & Secondary & 15 & 45.5 & 81 & 45.8 & 1.013 & $0.480-2.136$ & 0.974 \\
\hline & Tertiary & 8 & 24.2 & 41 & 23.1 & 0.942 & $0.395-2.247$ & 0.893 \\
\hline
\end{tabular}

Analysis of the social-demographic factors among the 210 pregnant mothers, 33 with UTI and 177 without: None of the factors was found to be associated with UTI, P $>0.05$. ${ }^{*}$ Nulliparous: those who have never delivered a baby before; Multiparous: those who have delivered more than one baby before; $1^{\text {st }}$ trimester: $1-3$ months; $2^{\text {nd }}$ trimester: 4 - 6 months; $3^{\text {rd }}$ trimester: 7 - 9 months; Primary class: 1 - 8; Secondary: form 1 - 4; Tertiary: post secondary education. 
Table 2. Lifestyle factors associated with UTI occurrence.

\begin{tabular}{|c|c|c|c|c|c|c|c|c|}
\hline \multirow{2}{*}{ Variables } & \multirow{2}{*}{ Response } & \multicolumn{2}{|c|}{ With UTI } & \multicolumn{2}{|c|}{ Without UTI } & \multirow{2}{*}{ OR } & \multirow{2}{*}{$95 \% \mathrm{CI}$} & \multirow{2}{*}{ P-value } \\
\hline & & $\mathbf{N}$ & $\%$ & $\mathrm{~N}$ & $\%$ & & & \\
\hline \multicolumn{2}{|c|}{ Overall } & 33 & 15.7 & 177 & 84.3 & & & \\
\hline \multirow{4}{*}{$\begin{array}{l}\text { Weekly Sexual } \\
\text { Intercourse }\end{array}$} & Once & 5 & 15.2 & 34 & 19.2 & 0.681 & $0.291-1.591$ & 0.373 \\
\hline & Twice & 10 & 30.3 & 69 & 39 & 2.282 & $1.073-4.854$ & 0.129 \\
\hline & Thrice & 14 & 42.4 & 56 & 31.6 & 0.628 & $0.294-1.342$ & 0.228 \\
\hline & $>3$ times & 4 & 12.1 & 18 & 10.2 & 0.821 & $0.259-2.601$ & 0.737 \\
\hline \multirow{2}{*}{$\begin{array}{c}\text { Number of Sexual } \\
\text { partners }\end{array}$} & One & 25 & 75.8 & 133 & 75.1 & 0.967 & $0.407-2.300$ & \multirow{2}{*}{0.139} \\
\hline & Multiple & 8 & 24.2 & 44 & 24.9 & 2.450 & $1.027-5.848$ & \\
\hline \multirow{2}{*}{$\begin{array}{c}\text { Material of } \\
\text { undergarment }\end{array}$} & Cotton & 10 & 30.3 & 76 & 42.9 & 1.731 & $0.778-3.851$ & \multirow{2}{*}{0.001} \\
\hline & Other than cotton & 23 & 69.7 & 101 & 57.1 & 3.051 & $1.556-5.981$ & \\
\hline \multirow{3}{*}{$\begin{array}{l}\text { Frequency of } \\
\text { changing } \\
\text { undergarment }\end{array}$} & Once & 9 & 27.3 & 36 & 20.3 & 2.282 & $0.291-1.591$ & 0.047 \\
\hline & Twice & 14 & 42.4 & 111 & 62.7 & 0.681 & $1.073-4.854$ & 0.029 \\
\hline & Thrice & 10 & 30.3 & 30 & 16.9 & 0.469 & $0.203-1.087$ & 0.043 \\
\hline
\end{tabular}

*Analysis of the lifestyle factors among the 210 pregnant mothers. Pregnant women with multiple (more than one) sexual partners and those who use undergarments made of materials other than cotton have higher risks of developing UTI than their counterparts.

contaminants based on CFU cut points revealed organisms similar to the pathogens isolated from UTI cases. The organisms were S. aureus (50\%), E. coli (30\%), $P$. aeroginosa and $K$. pneumoniae were at (10\%) each. E. coli isolates were evenly distributed across the different socio-demographics whereas $71.4 \%$ of $K$. pneumonia were from multiparous mothers with $80 \%$ of $S$. aureus isolates originating from mothers with multiple sexual partners. Regarding the Colony forming units (CFUs), S. aureus had the highest counts $\left(1.0 \times 10^{5}-1.0 \times 10^{7}\right)$ followed by E. coli $\left(1.0 \times 10^{5}-1.0 \times 10^{6}\right)$.

\subsection{Antimicrobial Susceptibility Profiles of Gram-Positive Isolates}

Comparative analysis revealed that the resistances exhibited by isolates whose counts fell below UTI cut off (contaminants) were similar to those observed among isolates recovered from UTI cases Table 3. None of the $S$. aureus strains recovered in this study was resistant to Amoxicillin-clavulanic acid, Nitrofurantoin, Chloramphenicol, Sulfamethoxazole/Trimethoprim, Linezolid, Quinupristin, and Ofloxacin indicating that these agents are still suitable for treatment of UTI. Resistance to Ceftazidime, Cefoxitin, Imipinem and Ciprofloxacin was observed in between $20 \%-40 \%$ of the $S$. aureus strains. Of the $S$. aureus isolates, $20 \%$ were MRSA while the prevalence of MDR among all Gram-positive strains was $25.8 \%$. Most of the MRSA strains were from multiparous mothers while a high proportion $(75 \%)$ of the MDR strains were from married mothers with tertiary level of education. Resistance of Enterococcus isolates to Cefoxitin, Erythromycin, Ampicillin and Ciprofloxacin was in the range of 30\% - 50\%, Table 3. 
Table 3. Resistance profiles of UTI isolates and contaminants.

\begin{tabular}{|c|c|c|c|c|c|c|c|c|c|c|c|c|c|c|c|c|c|c|c|c|c|}
\hline \multirow{2}{*}{ Species } & \multirow{2}{*}{$\begin{array}{l}\text { UTI Category } \\
\text { based on CFUs }\end{array}$} & \multicolumn{20}{|c|}{ Antimicrobials Resistance (\%) } \\
\hline & & $\mathbf{n}$ & AMP & AMC & $\mathrm{CAZ}$ & CR0 & CTX & FEP & FOX & IPM & NAL & CIP & SXT & r NIT & $\mathrm{CHL}$ & GEN & OFX & ERY & LNZ & QDA & DOX \\
\hline \multirow{2}{*}{ E. coli } & UTI & 45 & 89 & 13 & 49 & 49 & 62 & 28 & 15 & 0 & 86 & 62 & 86 & 2.2 & 31 & 63 & NT & NT & NT & NT & NT \\
\hline & Contaminant & 9 & 100 & 0 & 44 & 66 & 66 & 44 & 22 & 0 & 100 & 100 & 100 & 0 & 44 & 56 & NT & NT & NT & NT & NT \\
\hline \multirow{2}{*}{$K \cdot$ pneumoniae } & UTI & 21 & 100 & 76 & 100 & 100 & 100 & 38 & 85 & 14 & 100 & 100 & 100 & 66 & 5 & 98 & NT & NT & NT & NT & NT \\
\hline & Contaminant & 3 & 100 & 100 & 100 & 100 & 100 & 100 & 100 & 0 & 100 & 100 & 100 & 100 & 0 & 98 & NT & NT & NT & NT & NT \\
\hline \multirow[t]{2}{*}{ P. aeroginosa } & UTI & 6 & 100 & 100 & 0 & 100 & 100 & 0 & 100 & 0 & 100 & 0 & 100 & 100 & 100 & 100 & NT & NT & NT & NT & NT \\
\hline & Contaminant & 3 & 100 & 100 & 0 & 100 & 100 & 0 & 100 & 0 & 100 & 0 & 100 & 100 & 100 & 100 & NT & NT & NT & NT & NT \\
\hline Acinetobacter & UTI & 6 & 100 & 100 & 0 & 83 & 67 & 0 & 100 & 0 & 33 & 0 & 0 & 100 & 100 & 100 & NT & NT & NT & NT & NT \\
\hline \multirow[t]{2}{*}{ S. aureus } & UTI & 15 & 5100 & 0 & 40 & NT & NT & NT & 20 & 20 & 86 & 20 & 0 & 0 & 0 & 13 & 0 & 53 & 0 & 0 & 26 \\
\hline & Contaminant & 15 & 5100 & 0 & 86 & NT & NT & NT & 40 & 40 & 93 & 20 & 0 & 0 & 0 & 13 & 6.7 & 53 & 0 & 6.7 & 33 \\
\hline Enterococcus & UTI & 6 & 33 & 0 & 100 & NT & NT & NT & 50 & 100 & 100 & 33 & 17 & 0 & 17 & 17 & 0 & 50 & 0 & 17 & 0 \\
\hline
\end{tabular}

Antimicrobial susceptibility profiles of the UTI isolates and isolates whose counts fell below the $10^{5} \mathrm{CFU} / \mathrm{ml}$ UTI threshold (Contaminants). The panel of antimicrobials tested included the most common antimicrobials for UTIs and for treatment of Gram-negative and Gram-positive infections. ${ }^{*}$ AMP: Ampicillin; AMC: Amoxicillin-clavulanic; CAZ: Ceftazidime; CRO: Ceftriaxone; CTX: Cefotaxime; FEP: Cefepime; FOX: Cefoxitin; IPM: Imipinem; NAL: Nalidixic Acid; CIP: Ciprofloxacin; SXT: Sulfamethoxazole/Trimethoprim; NIT: Nitrofurantoin; CHL: Chloramphenicol; GEN: Gentamycin; OFX: Ofloxacin; ERY: Erythromycin; LNZ: Linezolid; QDA: Quinupristin; DOX: Doxycycline; NT: Not tested.

\subsection{Antimicrobial Susceptibility Profiles of Gram-Negative Isolates}

E. coli recorded higher resistances against Ampicillin (88.9\%), Nalidixic Acid (86.7\%) and Sulfamethoxazole/Trimethoprim (86.7\%). Resistance to third generation cephalosporins; Ceftazidime, Cefotaxime and Ceftriaxone was $48.9 \%$, $62.2 \%$ and $48.9 \%$ respectively. In contrast, Imipinem and Nitrofurantoin were effective against $100 \%$ and $98.8 \%$ of $E$. coli isolates respectively. A large proportion (86.6\%) of $E$. coli isolates were susceptible to Amoxicillin-Clavulanic acid.

All the Acinetobacter isolates on the other hand were resistant to Ampicillin, Amoxicillin-clavulanic acid, Cefoxitin, Nitrofurantoin and Chloramphenicol. None of the isolates was resistant to Ceftazidime, Cefepime, Ciprofloxacin and Sulfamethoxazole/Trimethoprim, this is in contrast to $E$. coli isolates that showed resistances in the range of $(28.9 \%$ - 62.2\%) against these antimicrobials.

All K. Pneumoniae and P. aeroginosa were resistant to Ampicillin, Ceftriaxone, Cefotaxime, Nalidixic Acid and Sulfamethoxazole/Trimethoprim. Resistance of $K$. pneumoniae against Chloramphenicol, Imipinem and Cefepime was $4.8 \%, 14.3 \%$ and $38.1 \%$ respectively with extreme resistance against Ciprofloxacin and Ceftazidime, Table 3.

Multi drug resistance (MDR), (resistance to $\geq 3$ classes of antimicrobials) was seen in $70.2 \%$ of Gram-negative isolates and $45 \%$ of these were isolated from multiparous mothers who used non-cotton undergarments and $15 \%$ among those with multiple sexual partners. Based on the resistance profiles, $22 \%$ of $E$. coli isolates and $16 \%$ of $K$. pneumonia isolates were ESBL-producers. This study found that majority of the ESBL producers exhibited co-resistance to multiple antimicrobials such as fluoroquinolones, aminoglycosides, Sulfamethoxazole- 
Trimethoprim and third generation cephalosporins. The most unique resistance phenotypes observed was combined resistance to $\beta$-lactams/Fluoroquinolones/ aminoglycosides like gentamicin and this was noted in $38.4 \%$ of Gram-negative bacteria.

\section{Discussion}

The overall prevalence of urinary tract infection in pregnant women in this study was $15.7 \%$ regardless of the women's age, parity and gestation. This falls within the global prevalence range of $13 \%-33 \%$ [3] and is comparable to the prevalence reported in Tanzania 15.5\% [4], but higher than those in studies from Ethiopia (10.4\%) and Uganda (13.3\%) [3] [14]. This was however lower than the prevalence of 31.3\% reported in Egypt [15]. Variations in prevalence rates from one country to another and among different regions of the continent can be attributed to environmental, economic and social habits of a community. In this study, knowledge of UTIs among the pregnant mothers was very low (37.6\%) and only $9 \%$, could link UTI acquisition to poor urogenital hygiene which was found to be a strongest predictor of these infections. This may partially fuel the high prevalence because fewer women seek treatment in time leading to poor treatment outcomes especially in well-established infections.

There was no significant association between UTI and maternal age, parity, occupation, stage of gestation, marital status or level of education making it comparable to other studies [4] [14]. However, maternal age and parity have previously been identified as risk factors for UTI among pregnant women [15]. Published studies indicates that simple hygiene habits such as voiding before and after sexual intercourse, use of absorbent cotton undergarments and wiping from anterior to posterior are advocated to decrease the risk of UTI [16]. Material of the undergarment and frequency of changing the undergarments were also found to be significantly contributing to the occurrence of UTI $(P<0.05)$, similar to a past study conducted in Egypt [15]. There is a possibility that the moisture trapped by non-cotton undergarments favor the proliferation of UTI agents including yeast hence increasing the chances of UTIs. Results from this study were in concordance with those from other studies that have reported that majority of the mothers who suffered UTI are in the second trimester. According to published studies, the risk of UTI begin at the $6^{\text {th }}$ week and the peak is usually around weeks 22 to 24 of gestation [5]. The high prevalence of UTIs in the second trimester can partially be attributed to the rapid changes in the physiology and immunology of pregnant women and due to the frequency of UTI tests during this phase.

From the foregoing, it is clear that treatment-seeking behavior and knowledge of UTIs may play a critical role as drivers for acquisition of UTIs. Having multiple sexual partners and frequency of sexual intercourse ( $\geq 2$ times/ week) had moderate association to UTI occurrence similar to findings from a study conducted in Egypt [15]. In general, UTIs are not considered as sexually transmitted 
infections (STIs) but this data suggest that males may be an important factor in the spread of UTIs between partners. Since males are normally asymptomatic for UTIs, there is a need to include both sexual partners for UTI diagnosis and treatment especially during pregnancy.

Gram-negative bacteria isolates were more prevalent (78.2\%) in UTIs than gram-positive bacteria (21.2\%). This could be due to the unique structure of gram negative bacteria which facilitates their attachment to the uroepithelial cells, and tissue invasion resulting in an invasive infection and pyelonephritis in pregnancy [17]. E. coli was the most predominant pathogen (44.5\%) similar to other findings in Tanzania, Ethiopia and Sudan [3] [4] [17]. K. pneumonia was the second most prevalent uropathogen similar to related studies in the neighboring Tanzania [4]. S. aureus was dominant at $15.1 \%$, but in general, the CFUs of this species were always more than those of any other species.

$\beta$-lactam antibiotics such as penicillins and cephalosporins, are considered safe during pregnancy and are usually prescribed for the treatment of UTIs during the gestational period [18]. All the gram-negative isolates except $A c i$ netobacter showed resistance in the range of $89 \%-100 \%$ to Ampicillin and Sulfamethoxazole-Trimethoprim (SXT). These results are similar to those published in two related studies in Kenya indicating that E. coli obtained in Kenya are more resistant to these antimicrobials than among similar isolates encountered in developed countries such as Japan [19]. This is particularly worrying because Sulfamethoxazole/Trimethoprim is heavily relied upon as a prophylaxis against opportunistic infections among individuals infected with HIV in Kenya. Resistance to Ciprofloxacin was in the range of $20 \%-100 \%$ depending with the species. This trend needs to be watched closely since Fluoroquinolones are thought to be the most effective antibiotic agents against $E$. coli infection [20]. In recent years, Kenya has reported development of resistance to Fluoroquinolones and extended-spectrum $\beta$-lactams in uropathogenic E. coli [21]. This continued increase in Fluoroquinolone resistance rates affects patient management and necessitates a change in the current treatment guidelines. An alarming finding in this study was the high prevalence of resistance to third generation Cephalosporins among the Gram-negative uropathogens. An earlier study done in Kenya reported similar resistance patterns in uropathogens [19]. This could be attributed to the presence of co-selection of genes conferring resistance to these antibiotics as well as issuance of prescriptions without susceptibility data. In this study, $14.3 \%$ of $K$. pneumonia, 20\% of $S$. aureus and all Enterococcus showed resistance to Imipinem. Although our sample size is rather small to determine national trends in resistance to Carbapenems, these findings are worrying considering that carbapenemase-producers in this study were recovered from otherwise asymptomatic participants. While this class of drugs remain useful for the treatment of serious infections, isolates resistant to carbapenems pose a serious challenge because none or few chemotherapeutic options remain for the seriously ill patient. The use of carbapenems as an alternative in developing coun- 
tries is also a challenge because few patients can afford this antimicrobial. Increased resistance to this class of antimicrobial will therefore lead to significant increase in mortality and morbidity of patients.

Compared to Gram-negative isolates, Gram-positive strains exhibited relatively lower resistances to a majority of antimicrobials tested in this study. Antimicrobial resistance patterns of isolates whose counts fell below the UTI threshold were similar to those of strains recovered from UTI-cut point specimen (cases). This confirms that indeed these isolates may be implicated in UTI and the low counts may partially be due to a receding or an establishing infection. If these "contaminants" represent a resistant population of uropathogens, they may become established and lead to serious and untreatable UTIs. Based on these results, it is therefore important to revise the existing diagnostic guidelines for UTIs. Further investigation based on genome analysis may in future reveal the genetic relatedness between these contaminants and the UTI isolates.

Multi drug resistance (MDR), was seen in in $96 \%$ of the isolated uropathogens, this is comparable to the findings in Ethiopia [3] and is indicative of very high resistance to commonly used antibiotics. This alarming phenomenon can be attributed to use of sub-optimal agents and inappropriate administration of antimicrobials in empiric therapies that can result in a shift to increased prevalence of resistant isolates in the community. Clinically, ESBLs limit the efficacy of $\beta$-lactams, including cephalosporins and are associated with high morbidity and mortality [12]. MRSA can easily spread within the community and cause multiple infections especially to persons who are immune-compromised. There is no doubt that the ESBLs and MRSA strains are playing a critical role as causes of UTIs in Kenya and this will pose a serious treatment challenge because of the ever-reducing treatment options. In the face of increasing resistance, there is a need to devise infection control methods that can reduce the incidences of UTI. Such measures will also include educating people, especially the most vulnerable groups (women) on predisposing and risk factors for UTI.

\section{Conclusion}

This study demonstrated that UTI is prevalent among antenatal mothers in Pumwani Maternity Hospital. Unsatisfactory personal hygiene practices had a significant role in developing UTI during pregnancy. Health education on personal hygiene practices should be emphasized by health care professionals during antenatal visits. Moderately high level of resistance against first line drugs and high level of resistance against $3^{\text {rd }}$ generation cephalosporins and fluoroquinolones was observed. There is therefore a need to revise existing empiric treatment regimens to periodically reflect prevailing resistance phenotypes based on our data. Isolates whose counts fall below the UTI threshold may actually be implicated in UTI and therefore this study recommends that urine culture and susceptibility testing should be an integral part of investigation in antenatal care guidelines. This study also recommends a review on the existing diagnostic 
guidelines based on CFUs. As antimicrobial resistance among bacterial pathogens is an evolving process, regular monitoring and surveillance is required to establish reliable information about resistance pattern of uropathogens for optimal empiric therapy of patients with UTI. This approach will help slow the emergence of resistance. This study forms a strong basis of future larger studies that should include whole genome analysis that can unravel transmission routes and molecular diversity of the UTI isolates.

\section{Acknowledgements}

The authors would like to thank and appreciate the study participants for their participation in the study. We thank KEMRI-CMR/Molecular Biology Laboratory for allowing us to conduct this study in your institution. Finally, we thank all the Students attached at the laboratory during the study duration and the entire CMR staffs. This work was partially supported by the National Research Fund (NRF) and AFRICA-AI-JAPAN Project funding.

\section{Conflict of Interests}

None.

\section{Author Contributions}

HO came up with the concept for the work and designed the study. She did the lab work, interpreted the data and drafted the manuscript. JM assisted in lab work. $\mathrm{CN}$ corrected the proposal, assisted in manuscript preparation and helped in its coordination. JK participated in the study design and helped in drafting the manuscript. All authors read and approved final manuscript.

\section{Authors' Information}

$\mathrm{HO}$ is a master's student in Kenya studying Infectious Diseases and Vaccinology. JM is a Medical Microbiology master's student. Dr. CN is a senior lecturer in Jomo Kenyatta University of agriculture and technology who has a vast experience in proposal development and microbial techniques. Dr. JK helped in actualizing the study and critically revising the content of the manuscript. He is a researcher in Centre of Microbiology Research, Kenya Medical Research Institute, Nairobi, Kenya.

\section{Ethical Approval}

Approved.

\section{References}

[1] Aseel, M., Meer, F.A., Kuwari, M.A. and Ismail, M. (2011) Prevalence and Predictors of Asymptomatic Bacteriuria among Pregnant Women Attending Primary Health Care in Qatar. Qatar National Research Strategy, 2011, 1715.

[2] Schnarr, J. and Smaill, F. (2008) Asymptomatic Bacteriuria and Symptomatic Uri- 
nary Tract Infections in Pregnancy. European Journal of Clinical Investigation, 38, 50-57.

[3] Agersew, A., Feleke, M., Yitayai, S., Ketema, T., Afework, K. and Abebe, A. (2012) Bacteria Profile and Drug Susceptibility Pattern of Urinary Tract Infection in Pregnant Women at University of Gonden Teaching Hospital, Northwest Ethiopia. BMC Research Notes, 5, 197-204. https://doi.org/10.1186/1756-0500-5-197

[4] Masinde, A., Gumodoka, B., Kilonzo, A. and Mshana, S.E. (2009) Prevalence of urinary Tract Infection among Pregnant Women at Bugando Medical Centre, Mwanza, Tanzania. Tanzania Journal of Health Research, 11, 154-159. https://doi.org/10.4314/thrb.v11i3.47704

[5] Delzell Jr., J.E. and Lefevre, M.L. (2000) Urinary Tract Infections during Pregnancy. American Family Physician, 61, 713-721.

[6] Gilbert, N.M., O’brien, V.P., Hultgren, S., Macones, G., Lewis, W.G. and Lewis, A.L. (2013) Urinary Tract Infection as a Preventable Cause of Pregnancy Complications: Opportunities, Challenges, and a Global Call to Action. Global Advances in Health and Medicine, 2, 59-69. https://doi.org/10.7453/gahmj.2013.061

[7] Kose, Y., Abasiyanik, M.F. and Salih, B.A. (2007) Antibiotic Resistance Rates of Escherichia Coli Urinary Tract Isolates in Rize Province, Turkey. The Journal of Infection in Developing Countries, 1, 147-150.

[8] Nicolle, L.E., Bradley, S., Colgan, R., Rice, J.C., Schaeffer, A. and Hooton, T.M. (2005) Infectious Diseases Society of America Guidelines for the Diagnosis and Treatment of Asymptomatic Bacteriuria in Adults. Clinical Infectious Diseases, 40, 643-54. https://doi.org/10.1086/427507

[9] Monica, C. (2000) Biochemical Tests to Identify Bacteria. Cambridge University Press.

[10] Bauer, A.W., Kirby, W.M.M., Sherris, J.C. and Turck, M. (1996) Antibiotic Susceptibility Testing by a Standardized Single Disk Method. American Journal of Clinical Pathology, 45, 493-496.

[11] Clinical Laboratory Standard Institute (CLSI) (2016) Performance Standards for Antimicrobial Susceptibility Testing; Twenty-Sixth Informational Supplement. Vol. CLSI Docum, No. Wayne, PA.

[12] Rawat, D. and Nair, D. (2010) Extended-Spectrum $\beta$-Lactamases in Gram Negative Bacteria. Journal of Global Infectious Diseases, 2, 263-274. https://doi.org/10.4103/0974-777X.68531

[13] Jain, A., Agarwal, A. and Verma, R.K. (2008) Cefoxitin Disc Diffusion Test for Detection of Meticillin-Resistant Staphylococci. Journal of Medical Microbiology, 57, 957-961. https://doi.org/10.1099/jmm.0.47152-0

[14] Andabati, G. and Byamugisha, J. (2010) Microbial Aetiology and Sensitivity of Asymptomatic Bacteriuria among Ante-Natal Mothers in Mulago Hospital, Uganda. African Health Sciences, 10, 349-352.

[15] Dimetry, S.R., El-tokhy, H.M., Abdo, N.M., Ebrahim, M.A. and Eissa, M. (2007) Urinary Tract Infection and Adverse Outcome of Pregnancy Clinical Pathology Department. The Journal of the Egyptian Public Health Association, 82, 203-218.

[16] Griebling, T.L. (2007) Urinary Tract Infection in Women. In: Litwin, M.S. and Saigal, C.S., Eds., Urologic Diseases in America, US Department of Health and Human Services, Public Health Service, National Institutes of Health, National Institute of Diabetes and Digestive and Kidney Diseases, US Government Printing Office: NIH Publication, Washington DC, 589-619.

[17] Hamdan, H.Z., Ziad, A.H.M., Ali, S.K. and Adam, I. (2011) Epidemiology of Uri- 
nary Tract Infections and Antibiotics Sensitivity among Pregnant Women at Khartoum North Hospital. Annals of Clinical Microbiology and Antimicrobials, 10, 2. https://doi.org/10.1186/1476-0711-10-2

[18] Guinto, V.T., De Guia, B., Festin, M.R. and Dowswell, T. (2010) Different Antibiotic Regimens for Treating Asymptomatic Bacteriuria in Pregnancy. The Cochrane Database of Systematic Reviews, No. 9, CD007855.

[19] Ayoyi, A.O., Kikuvi, G., Bii, C. and Kariuki, S. (2017) Prevalence, Aetiology and Antibiotic Sensitivity Profile of Asymptomatic Bacteriuria Isolates from Pregnant Women in Selected Antenatal Clinic from Nairobi, Kenya. Pan African Medical Journal, 26, 1-12. https://doi.org/10.11604/pamj.2017.26.41.10975

[20] Oladeinde, B.H., Omoregie, R. and Oladeinde, O.B. (2015) Asymptomatic Urinary Tract Infection among Pregnant Women Receiving Ante-Natal Care in a Traditional Birth Home in Benin City, Nigeria. Ethiopian Journal of Health Sciences, 15, 3-8.

[21] Kariuki, S., et al. (2007) Escherichia Coli from Community-Acquired Urinary Tract Infections Resistant to Fluoroquinolones and Extended-Spectrum Beta-Lactams. The Journal of Infection in Developing Countries, 1, 257-262. 